\title{
Linear Diophantine equations in Piatetski-Shapiro sequences
}

\author{
by \\ Toshiki Matsusaka and Kota Saito (Nagoya)
}

1. Introduction. Let $\lfloor x\rfloor$ denote the integer part of $x \in \mathbb{R}$. For a non-integral $\alpha>0$, the sequence $\left(\left\lfloor n^{\alpha}\right\rfloor\right)_{n=1}^{\infty}$ is called the Piatetski-Shapiro sequence with exponent $\alpha$. Let $\operatorname{PS}(\alpha)=\left\{\left\lfloor n^{\alpha}\right\rfloor: n \in \mathbb{N}\right\}$. We say that an equation $f\left(x_{1}, \ldots, x_{n}\right)=0$ is solvable in $\operatorname{PS}(\alpha)$ if there are infinitely many pairwise distinct tuples $\left(x_{1}, \ldots, x_{n}\right) \in \operatorname{PS}(\alpha)^{n}$ satisfying this equation. In this article, we investigate the solvability in $\operatorname{PS}(\alpha)$ of linear Diophantine equations

$$
a x+b y=c z
$$

for all fixed $a, b, c \in \mathbb{N}$. For example, the solvability of the equation $y=\theta x+\eta$ for $\theta, \eta \in \mathbb{R}$ with $\theta \notin\{0,1\}$ has been studied by Glasscock Gla17, Gla20]. He asserts that if the equation $y=\theta x+\eta$ has infinitely many solutions $(x, y) \in \mathbb{N}^{2}$, then for Lebesgue-a.e. $\alpha>1$ it is solvable or not in $\operatorname{PS}(\alpha)$ according as $\alpha<2$ or $\alpha>2$. As a direct consequence, for Lebesgue-a.e. $1<\alpha<2$, the equation $z=(a / c) x+(b / c)$ is solvable in $\operatorname{PS}(\alpha)$ for all $a, b, c \in \mathbb{N}$ with $\operatorname{gcd}(a, c) \mid b$. In other words, the equation (1.1) with $\operatorname{gcd}(a, c) \mid b$ is solvable in $\operatorname{PS}(\alpha)$. On the other hand, for $\alpha>2$, we did not know at all whether the equation (1.1) is solvable in $\operatorname{PS}(\alpha)$ or not.

Our main result provides an answer to this question. We consider the set of $\alpha$ in a short interval $[s, t] \subset(2, \infty)$ such that $(1.1)$ is solvable. The following theorem asserts that the Hausdorff dimension of this set is positive. To state the theorem, let $\{x\}$ be the fractional part of $x \in \mathbb{R}$, and $\operatorname{dim}_{\mathrm{H}}(X)$ the Hausdorff dimension of $X \subseteq \mathbb{R}$ (the definition will be recalled in Section 2).

2020 Mathematics Subject Classification: Primary 11K55; Secondary 11D04.

Key words and phrases: Piatetski-Shapiro sequence, Hausdorff dimension, Diophantine equation, arithmetic progression, discrepancy.

Received 27 September 2020; revised 23 January 2021.

Published online 10 May 2021. 
Theorem 1.1. Let $a, b, c \in \mathbb{N}$. For all positive real numbers $2<s<t$, $\operatorname{dim}_{\mathrm{H}}(\{\alpha \in[s, t]: a x+b y=c z$ is solvable in $\operatorname{PS}(\alpha)\})$

$$
\geq\left\{\begin{array}{l}
\left(s+\frac{s^{3}}{\left(2+\{s\}-2^{1-\lfloor s\rfloor}\right)(2-\{s\})}\right)^{-1} \quad \text { if } a=b=c, \\
2\left(s+\frac{s^{3}}{\left(2+\{s\}-2^{1-\lfloor s\rfloor}\right)(2-\{s\})}\right)^{-1} \quad \text { otherwise. }
\end{array}\right.
$$

Note that the lower bound in either case is greater than $1 / s^{3}$ for all $2<s<t$. The positivity of the Hausdorff dimension implies that this set is uncountable for any closed interval $[s, t] \subset(2, \infty)$. Moreover, we can easily prove the following:

Corollary 1.2. For any closed interval $I \subset(2, \infty)$, the set of $\alpha \in I$ such that $a x+b y=c z$ is solvable in $\operatorname{PS}(\alpha)$ is uncountable and dense in $I$.

In particular, for $a=b=1, c=2$, a pairwise distinct tuple $(x, z, y)$ satisfying (1.1) forms an arithmetic progression of length 3 . Therefore Corollary 1.2 implies

Corollary 1.3. For any closed interval $I \subset(2, \infty)$, the set of $\alpha \in I$ such that $\mathrm{PS}(\alpha)$ contains infinitely many arithmetic progressions of length 3 is uncountable and dense in $I$.

There are some related works on arithmetic progressions and PiatetskiShapiro sequences. It is an exercise to show that for all $1<\alpha<2$, the set $\operatorname{PS}(\alpha)$ contains arbitrarily long arithmetic progressions (consisting of consecutive elements). Frantzikinakis and Wierdl FW09 proved that any set of positive integers with positive upper density contains arbitrarily long arithmetic progressions whose common difference belongs to $\operatorname{PS}(\alpha)$ for all non-integral $\alpha>1$ (here we say that $A \subseteq \mathbb{N}$ has positive upper density if $\left.\varlimsup_{N \rightarrow \infty}|A \cap\{1, \ldots, N\}| / N>0\right)$. This result is an extension of Szemerédi's theorem [Sze75]. Furthermore, the second author and Yoshida [SY19] gave another extension of Szemerédi's theorem to Piatetski-Shapiro sequences by showing that for any $A \subseteq \mathbb{N}$ with positive upper density, the set $\left\{\left\lfloor n^{\alpha}\right\rfloor\right.$ : $n \in A\}$ with $1<\alpha<2$ contains arbitrarily long arithmetic progressions. They also posed a question:

Question 1.4 ([SY19, Question 13]). Is it true that $\sup \{\alpha \geq 1: \operatorname{PS}(\alpha)$ contains arbitrarily long arithmetic progressions $\}=2$ ?

We do not get any answer to this question here, but surprisingly, by Corollary 1.3 , the supremum of $\alpha$ such that $\operatorname{PS}(\alpha)$ contains infinitely many arithmetic progressions of length 3 is positive infinity. Glasscock also posed a related question for the equation (1.1) with $a=b=c=1$. 
Question 1.5 ([Gla17, Question 6]). Does there exist an $\alpha_{S}>1$ with the property that for Lebesgue-a.e. or all $\alpha>1$, the equation $x+y=z$ is solvable or not in $\operatorname{PS}(\alpha)$ according as $\alpha<\alpha_{S}$ or $\alpha>\alpha_{S}$ ?

By Corollary 1.2, the case with "all $\alpha>1$ " in Question 1.5 is false since the supremum of $\alpha>0$ such that (1.1) is solvable in $\operatorname{PS}(\alpha)$ is positive infinity. However, the case with "Lebesgue-a.e." in Question 1.5 is still open.

The rest of the article is organized as follows. First in Section 2 we define the discrepancy of the sequences and the Hausdorff dimension, and describe some known useful results. In Sections 3 and 4 , we prove a series of lemmas. Finally we provide a proof of Theorem 1.1.

Notation. Let $\mathbb{N}=\{1,2, \ldots\}$. For $x \in \mathbb{R}$, let $\lfloor x\rfloor$ denote the integer part of $x,\{x\}$ denote the fractional part of $x$, and $\lceil x\rceil$ denote the minimum integer $n$ such that $x \leq n$. A tuple $\left(x_{1}, \ldots, x_{k}\right) \in \mathbb{R}^{k}$ is called pairwise distinct if $\#\left\{x_{1}, \ldots, x_{k}\right\}=k$. Let $\sqrt{-1}$ denote the imaginary unit, and define $e(x)$ by $e^{2 \pi \sqrt{-1} x}$ for all $x \in \mathbb{R}$.

2. Preparations. For all $\mathbf{x}=\left(x_{1}, \ldots, x_{d}\right) \in \mathbb{R}^{d}$, define

$$
\{\mathbf{x}\}=\left(\left\{x_{1}\right\}, \ldots,\left\{x_{d}\right\}\right) .
$$

Let $\left(\mathbf{x}_{n}\right)_{1 \leq n \leq N}$ be a sequence composed of $\mathbf{x}_{n} \in \mathbb{R}^{d}$ for all $1 \leq n \leq N$. We define the discrepancy $D\left(\mathbf{x}_{1}, \ldots, \mathbf{x}_{N}\right)$ of $\left(\mathbf{x}_{n}\right)_{n=1}^{N}$ by

$$
\sup _{\substack{0 \leq a_{i}<b_{i} \leq 1 \\ 1 \leq i \leq d}}\left|\frac{\#\left\{n \in \mathbb{N} \cap[1, N]:\left\{\mathbf{x}_{n}\right\} \in \prod_{i=1}^{d}\left[a_{i}, b_{i}\right)\right\}}{N}-\prod_{i=1}^{d}\left(b_{i}-a_{i}\right)\right| .
$$

In order to evaluate an upper bound for the discrepancy, we use the following inequality which was shown by Koksma [Kok50] and Szüsz [Szü52] independently: there exists $C_{d}>0$ which depends only on $d$ such that for all $K \in \mathbb{N}$, we have

$$
D\left(\mathbf{x}_{1}, \ldots, \mathbf{x}_{N}\right) \leq C_{d}\left(\frac{1}{K}+\sum_{\substack{0<\|\mathbf{k}\|_{\infty} \leq K \\ \mathbf{k} \in \mathbb{Z}^{d}}} \frac{1}{\nu(\mathbf{k})}\left|\frac{1}{N} \sum_{n=1}^{N} e^{2 \pi \sqrt{-1}\left\langle\mathbf{k}, \mathbf{x}_{n}\right\rangle}\right|\right),
$$

where we let $\langle\cdot, \cdot\rangle$ denote the standard inner product and define

$$
\|\mathbf{k}\|_{\infty}=\max \left(\left|k_{1}\right|, \ldots,\left|k_{d}\right|\right), \quad \nu(\mathbf{k})=\prod_{i=1}^{d} \max \left(1,\left|k_{i}\right|\right) .
$$

This inequality is sometimes reffered as the Erdôs-Turán-Koksma inequality. We refer the readers to [DT97, Theorem 1.21] for more details on discrepancies and a proof of (2.1). This inequality reduces the estimate of the 
discrepancy to that of exponential sums. Furthermore, the exponential sum is evaluated by the following lemma.

Lemma 2.1 (van der Corput's $k$ th derivative test). Let $f(x)$ be real and have continuous derivatives up to $k$ th order, where $k \geq 4$. Let $\lambda_{k} \leq f^{(k)}(x) \leq$ $h \lambda_{k}$ (or the same for $\left.-f^{(k)}(x)\right)$. Let $b-a \geq 1$. Then there exists $C(h, k)>0$ such that

$$
\left|\sum_{a<n \leq b} e^{2 \pi \sqrt{-1} f(n)}\right| \leq C(h, k)\left((b-a) \lambda_{k}^{1 /\left(2^{k}-2\right)}+(b-a)^{1-2^{2-k}} \lambda_{k}^{-1 /\left(2^{k}-2\right)}\right) .
$$

Proof. See Titchmarsh's book [Tit86, Theorem 5.13].

We next introduce the Hausdorff dimension. For every $U \subseteq \mathbb{R}$, write the diameter of $U$ as $\operatorname{diam}(U)=\sup _{x, y \in U}|x-y|$. Fix $\delta>0$. For all $F \subseteq \mathbb{R}$ and $s \in[0,1]$, we define

$$
\mathcal{H}_{\delta}^{s}(F)=\inf \left\{\sum_{j=1}^{\infty} \operatorname{diam}\left(U_{j}\right)^{s}: F \subseteq \bigcup_{j=1}^{\infty} U_{j},(\forall j \in \mathbb{N}) \operatorname{diam}\left(U_{j}\right) \leq \delta\right\},
$$

and $\mathcal{H}^{s}(F)=\lim _{\delta \rightarrow+0} \mathcal{H}_{\delta}^{s}(F)$ is called the $s$-dimensional Hausdorff measure of $F$. Further,

$$
\operatorname{dim}_{\mathrm{H}}(F)=\inf \left\{s \in[0,1]: \mathcal{H}^{s}(F)=0\right\}
$$

is called the Hausdorff dimension of $F$. Note that the Hausdorff dimension can be defined on all metric spaces, but we use only $\mathbb{R}$ in this article. By the definition, the following basic properties hold:

- (Monotonicity) for all $F \subseteq E \subseteq \mathbb{R}$, we have $\operatorname{dim}_{\mathrm{H}}(F) \leq \operatorname{dim}_{\mathrm{H}}(E)$;

- (Countable stability) if $F_{1}, F_{2}, \ldots \subseteq \mathbb{R}$ is a countable sequence of sets, then $\operatorname{dim}_{H}\left(\bigcup_{n=1}^{\infty} F_{n}\right)=\sup _{n \in \mathbb{N}} \operatorname{dim}_{\mathrm{H}}\left(F_{n}\right)$.

We refer the readers to Falconer's book Fal14 for more details on fractal dimensions. In order to prove Theorem 1.1, we construct a general Cantor set which is a subset of the set of all $\alpha$ such that (1.1) is solvable in $\operatorname{PS}(\alpha)$. In [Fal14, (4.3)], we can see a general construction of Cantor sets and a technique to evaluate their Hausdorff dimension as follows: Let $[0,1]=E_{0} \supseteq E_{1} \supseteq \cdots$ be a decreasing sequence of sets, with each $E_{k}$ a union of a finite number of disjoint closed intervals called $k$ th level basic intervals, with each interval of $E_{k}$ containing at least two intervals of $E_{k+1}$, and with the maximum length of $k$ th level intervals tending to 0 as $k \rightarrow \infty$. Then let

$$
F=\bigcap_{k=0}^{\infty} E_{k}
$$

Lemma 2.2 ([Fal14, Example 4.6(a)]). Suppose in the general construction (2.2) each $(k-1)$ st level interval contains at least $m_{k} \geq 2 k$ th level 
intervals $(k=1,2, \ldots)$ which are separated by gaps of at least $\delta_{k}$, where $0<\delta_{k+1}<\delta_{k}$ for each $k$. Then

$$
\operatorname{dim}_{\mathrm{H}}(F) \geq \varliminf_{k \rightarrow \infty} \frac{\log \left(m_{1} \cdots m_{k-1}\right)}{-\log \left(m_{k} \delta_{k}\right)} .
$$

Since the Hausdorff dimension is stable under similarity transformations, the initial interval $E_{0}$ may be taken to be an arbitrary closed interval. Moreover, let $E_{k}^{\circ}$ be the set of interior points of $E_{k}$ for all $k \in \mathbb{N}$. Then the Hausdorff dimension of $\bigcap_{k=0}^{\infty} E_{k}^{\circ}$ is equal to that of $\bigcap_{k=0}^{\infty} E_{k}$. To see why, let $\mathcal{N}_{k}$ be the boundary of $E_{k}$, that is, the set of all end points of $k$ th level intervals. We easily see that

$$
\mathcal{N}:=F \backslash \bigcap_{k=0}^{\infty} E_{k}^{\circ} \subset \bigcup_{k=0}^{\infty} \mathcal{N}_{k}=: \mathcal{N}_{\infty}
$$

Since each $\mathcal{N}_{k}$ is a finite set, $\mathcal{N}_{\infty}$ is countable. By monotonicity, and the fact that the Hausdorff dimension of a countable set is 0 , we get

$$
0 \leq \operatorname{dim}_{\mathrm{H}}(\mathcal{N}) \leq \operatorname{dim}_{\mathrm{H}}\left(\mathcal{N}_{\infty}\right)=0,
$$

that is, $\operatorname{dim}_{\mathrm{H}}(\mathcal{N})=0$. Therefore by countable stability,

$$
\operatorname{dim}_{\mathrm{H}}(F)=\max \left\{\operatorname{dim}_{\mathrm{H}}\left(\bigcap_{k=0}^{\infty} E_{k}^{\circ}\right), \operatorname{dim}_{\mathrm{H}}(\mathcal{N})\right\}=\operatorname{dim}_{\mathrm{H}}\left(\bigcap_{k=0}^{\infty} E_{k}^{\circ}\right) .
$$

To summarize this discussion, we have the following:

LEMmA 2.3. Let $E_{0}$ be any open interval, and let $E_{0} \supseteq E_{1} \supseteq \cdots$ be a decreasing sequence of sets, with each $E_{k}$ a union of a finite number of disjoint open intervals, and with the maximum length of kth level intervals tending to 0 as $k \rightarrow \infty$. Suppose each $(k-1)$ st level interval contains at least $m_{k} \geq 2$ kth level intervals $(k=1,2, \ldots)$ which are separated by gaps of at least $\delta_{k}$, where $0<\delta_{k+1}<\delta_{k}$ for each $k$. Then

$$
\operatorname{dim}_{\mathrm{H}}\left(\bigcap_{k=0}^{\infty} E_{k}\right) \geq \underset{k \rightarrow \infty}{\lim } \frac{\log \left(m_{1} \cdots m_{k-1}\right)}{-\log \left(m_{k} \delta_{k}\right)}
$$

3. Lemmas I. We write $O(1)$ for a bounded quantity. If this bound depends only on some parameters $a_{1}, \ldots, a_{n}$, then for instance we write $O_{a_{1}, \ldots, a_{n}}(1)$. As is customary, we often abbreviate $O(1) X$ and $O_{a_{1}, \ldots, a_{n}}(1) X$ to $O(X)$ and $O_{a_{1}, \ldots, a_{n}}(X)$ respectively for a non-negative quantity $X$. We also write $f(X) \ll g(X)$ and $f(X) \ll_{a_{1}, \ldots, a_{n}} g(X)$ if $f(X)=O(g(X))$ and $f(X)=O_{a_{1}, \ldots, a_{n}}(g(X))$ respectively, where $g(X)$ is non-negative.

Let us consider the solvability of the equation (1.1). In this and subsequent sections, we fix $a, b, c, d \in \mathbb{N}$ with $d \geq 2$ and $\beta, \gamma \in \mathbb{R}$ with $d<\beta<$ $\gamma<d+1$. Unless it causes confusion, we do not indicate their dependence 
hereafter. Take a large parameter $x_{0}=x_{0}(a, b, c, d, \beta, \gamma)>0$. For all integers $x \geq x_{0}$, we define

$$
J_{a, b, c}(x)= \begin{cases}\left(\left(\frac{b}{c x^{2} \log x}+\frac{a}{c}\right)^{1 / \gamma} x,\left(\frac{a}{c}\right)^{1 / \beta} x\right)_{\mathbb{N}} \backslash x \mathbb{N} & \text { if } c<a, \\ \left(\left(\frac{a}{c-b\left(x^{2} \log x\right)^{-1}}\right)^{1 / \beta} x,\left(\frac{a}{c}\right)^{1 / \gamma} x\right)_{\mathbb{N}} & \text { if } a<c, \\ \left(2^{1 / \gamma}\left(x+\frac{1}{x\lceil\log x\rceil}\right), 2^{1 / \beta} x\right)_{\mathbb{N}} & \text { if } a=b=c,\end{cases}
$$

where we let $(s, t)_{\mathbb{N}}$ denote $(s, t) \cap \mathbb{N}$, and set $x \mathbb{N}=\{x n: n \in \mathbb{N}\}$. Note that $J_{a, b, c}(x)$ is non-empty if $x_{0}$ is sufficiently large. When $a=c$ and $b \neq c$, $J_{a, b, c}(x)$ is not defined above, but this case comes down to the case when $a \neq c$ by switching the roles of $(a, x)$ and $(b, y)$. Thus the three cases in the definition of $J_{a, b, c}(x)$ are sufficient.

Lemma 3.1. Assume that $a \neq c$. Then there exists $C>0$ such that for all integers $x \geq x_{0}$ and for all $z \in J_{a, b, c}(x)$, we can find $\alpha=\alpha(x, z) \in(\beta, \gamma)$ such that $a x^{\alpha}+b=c z^{\alpha}$, and

$$
\left|\alpha-\frac{\log (a / c)}{\log (z / x)}\right| \leq \frac{C}{x^{2} \log x} .
$$

Proof. Fix any $x \geq x_{0}$ and $z \in J_{a, b, c}(x)$. For all $u \in \mathbb{R}$, consider the continuous function $f(u)=a x^{u}+b-c z^{u}$. We consider two cases.

CASE $a>c$. Let

$$
\alpha_{0}=\frac{\log (a / c)}{\log (z / x)}, \quad \alpha_{1}=\frac{\log \left(a / c+b /\left(c x^{2} \log x\right)\right)}{\log (z / x)} .
$$

Then $z \in J_{a, b, c}(x)$ implies $\beta<\alpha_{0}<\alpha_{1}<\gamma$. It follows that $f\left(\alpha_{0}\right)=b>0$. By taking a larger $x_{0}$ if necessary, we have

$$
f\left(\alpha_{1}\right)=x^{\alpha_{1}}\left(a+b x^{-\alpha_{1}}-c(z / x)^{\alpha_{1}}\right) \leq x^{\alpha_{1}}\left(a+b /\left(x^{2} \log x\right)-c(z / x)^{\alpha_{1}}\right)=0 .
$$

Therefore, by the intermediate value theorem, there exists a zero $\alpha=\alpha(x, z)$ of $f$ such that $\beta<\alpha_{0} \leq \alpha \leq \alpha_{1}<\gamma$. Since $\log (1+u) \leq u$ for all $u \in(-1, \infty)$, we have

$$
\left|\alpha_{1}-\alpha_{0}\right|=\frac{\log \left(1+b /\left(a x^{2} \log x\right)\right)}{\log (z / x)} \leq \frac{b}{a x^{2} \log x} \cdot \frac{1}{\log (z / x)} .
$$

From this inequality and $1 / \log (z / x) \ll_{a, c, \gamma} 1$, we obtain (3.1).

CASE $c>a$. Let

$$
\alpha_{0}=\frac{\log (c / a)}{\log (x / z)}, \quad \alpha_{1}^{\prime}=\frac{\log \left(c / a-b /\left(a x^{2} \log x\right)\right)}{\log (x / z)} .
$$


Since $z \in J_{a, b, c}(x)$, we have $\beta<\alpha_{1}^{\prime}<\alpha_{0}<\gamma$ and $x \ll_{a, b, c, \beta, \gamma} z$. Then by the calculation in Case $a>c, f\left(\alpha_{0}\right)=b>0$. Further, $x \ll z$ implies $z^{-\alpha_{1}^{\prime}} \leq$ $z^{-\beta} \ll x^{-\beta}$. Thus if $x_{0}$ is sufficiently large, we have $z^{-\alpha_{1}^{\prime}} \leq 1 /\left(x^{2} \log x\right)$, which yields

$$
f\left(\alpha_{1}^{\prime}\right)=z^{\alpha_{1}^{\prime}}\left(a(x / z)^{\alpha_{1}^{\prime}}+b z^{-\alpha_{1}^{\prime}}-c\right) \leq z^{\alpha_{1}^{\prime}}\left(a(x / z)^{\alpha_{1}^{\prime}}+b /\left(x^{2} \log x\right)-c\right)=0 .
$$

Therefore, by the intermediate value theorem, there exists a zero $\alpha=\alpha(x, z)$ of $f$ such that $\beta<\alpha_{1}^{\prime} \leq \alpha \leq \alpha_{0}<\gamma$. Since $|\log (1-u)| \leq 2 u$ for all $u \in(0,1 / 2)$, we have

$$
\left|\alpha_{0}-\alpha_{1}^{\prime}\right|=\frac{\left|\log \left(1-b /\left(c x^{2} \log x\right)\right)\right|}{\log (x / z)} \leq \frac{2 b}{c x^{2} \log x} \cdot \frac{1}{\log (x / z)}
$$

provided $x_{0}$ is sufficiently large. From this inequality and $1 / \log (x / z) \ll_{a, c, \gamma} 1$, we obtain 3.1.

Lemma 3.2. There exists $C>0$ such that for all integers $x \geq x_{0}$ and $z \in$ $J_{1,1,1}(x)$, we can find $\alpha=\alpha(x, z) \in(\beta, \gamma)$ such that $x^{\alpha}+\left(x+(x\lceil\log x\rceil)^{-1}\right)^{\alpha}$ $=z^{\alpha}$, and

$$
\left|\alpha-\frac{\log 2}{\log (z / x)}\right| \leq \frac{C}{x^{2} \log x} .
$$

Proof. Take any $x \geq x_{0}$ and $z \in J_{1,1,1}(x)$. For all $u \in \mathbb{R}$, consider the continuous function $f(u)=x^{u}+\left(x+(x\lceil\log x\rceil)^{-1}\right)^{u}-z^{u}$, and set

$$
\alpha_{0}=\frac{\log 2}{\log (z / x)}, \quad \alpha_{1}=\frac{\log 2}{\log \left(\frac{z}{x+(x\lceil\log x\rceil)^{-1}}\right)} .
$$

By $z \in J_{1,1,1}(x)$, we get $\beta<\alpha_{0}<\alpha_{1}<\gamma$. By the definitions of $\alpha_{0}$ and $\alpha_{1}$, we have

$$
f\left(\alpha_{0}\right)>z^{\alpha_{0}}\left(\frac{1}{2}+\frac{1}{2}-1\right)=0, \quad f\left(\alpha_{1}\right)<z^{\alpha_{1}}\left(\frac{1}{2}+\frac{1}{2}-1\right)=0 .
$$

Therefore, by the intermediate value theorem, there exists a zero $\alpha=\alpha(x, z)$ of $f$ such that $\alpha_{0} \leq \alpha \leq \alpha_{1}$. Further, we deduce $(3.2)$ since

$$
\left|\alpha_{1}-\alpha_{0}\right| \leq \frac{\gamma^{2}}{\log 2} \log \left(1+\frac{1}{x^{2} \log x}\right) \leq \frac{\gamma^{2}}{\log 2} \cdot \frac{1}{x^{2} \log x}
$$

LEMMA 3.3. Let $\varepsilon>0$ be an arbitrarily small real number. For all $X, Y, Z \in \mathbb{N}$, and $\alpha \in \mathbb{R}$ with $\beta<\alpha<\gamma$, if

$$
a X^{\alpha}+b Y^{\alpha}=c Z^{\alpha}
$$

then there exists $n_{0} \in \mathbb{N}$ such that

$$
\begin{aligned}
& a\left\lfloor\left(n_{0} X\right)^{\alpha}\right\rfloor+b\left\lfloor\left(n_{0} Y\right)^{\alpha}\right\rfloor=c\left\lfloor\left(n_{0} Z\right)^{\alpha}\right\rfloor, \\
& \max \left(\left\{\left(n_{0} X\right)^{\alpha}\right\},\left\{\left(n_{0} Y\right)^{\alpha}\right\},\left\{\left(n_{0} Z\right)^{\alpha}\right\}\right)<1 / 2,
\end{aligned}
$$




$$
n_{0} \ll_{\varepsilon}(X+Y)^{\gamma^{2} /\left(\left(2+\{\beta\}-2^{1-\lfloor\beta\rfloor}\right)(2-\{\gamma\})\right)+\varepsilon} .
$$

Proof. Choose $X, Y, Z \in \mathbb{N}$ and $\alpha$ with $\beta<\alpha<\gamma$ satisfying (3.3). For all $n \in \mathbb{N}$,

$$
c\left\lfloor(n Z)^{\alpha}\right\rfloor=c(n Z)^{\alpha}-c\left\{(n Z)^{\alpha}\right\}=a\left\lfloor(n X)^{\alpha}\right\rfloor+b\left\lfloor(n Y)^{\alpha}\right\rfloor+\delta(n),
$$

where we define $\delta(n)=a\left\{(n X)^{\alpha}\right\}+b\left\{(n Y)^{\alpha}\right\}-c\left\{(n Z)^{\alpha}\right\}$. Let

$$
A=\left\{n \in \mathbb{N}:|\delta(n)|<1, \max \left(\left\{(n X)^{\alpha}\right\},\left\{(n Y)^{\alpha}\right\},\left\{(n Z)^{\alpha}\right\}\right)<1 / 2\right\},
$$

and note that any $n \in A$ satisfies (3.4) and (3.5). Let us show the existence of $n \in A$ satisfying (3.6). Take a small $\xi=\xi(d, \beta, \gamma, \varepsilon)>0$ and take a sufficiently large parameter $R=R(a, b, c, d, \beta, \gamma, \varepsilon)$. Set

$$
N=\left\lceil R(X+Y)^{\gamma^{2} /\left(\left(2+\{\beta\}-2^{1-\lfloor\beta\rfloor}\right)(2-\{\gamma\})\right)+\varepsilon}\right\rceil,
$$

and set $\psi=\{\beta\}-2+\left(2^{d+2}-2\right)\left(1 / 2^{d}-2 \xi\right)$. Since

$$
\psi=2+\{\beta\}-2^{1-\lfloor\beta\rfloor}+O(\xi),
$$

we have $0<\psi<\beta<\alpha$ for $\xi$ small enough. Moreover, we let $L\left(h_{1}, h_{2}\right)=$ $\left(h_{1} X^{\alpha}+h_{2} Y^{\alpha}\right) / c$.

Case 1. We first discuss the case when

$$
\left|L\left(h_{1}, h_{2}\right)\right| \geq N^{-\psi}
$$

for all $h_{1}, h_{2} \in \mathbb{Z}$ with $0<\max \left(\left|h_{1}\right|,\left|h_{2}\right|\right) \leq N^{\xi}$. In this case, define

$$
A_{1}=\left\{n \in \mathbb{N}: 0 \leq\left\{(n X)^{\alpha} / c\right\}<\frac{1}{4 a c}, 0 \leq\left\{(n Y)^{\alpha} / c\right\}<\frac{1}{4 b c}\right\} .
$$

Then we have $A_{1} \subseteq A$. Indeed, take any $n \in A_{1}$. We see that

$$
(n X)^{\alpha}=c\left\lfloor(n X)^{\alpha} / c\right\rfloor+c\left\{(n X)^{\alpha} / c\right\} .
$$

Since the first term on the right-hand side of (3.11) is an integer and the second term belongs to $[0,1)$ by $n \in A_{1}$, we get $\left\{(n X)^{\alpha}\right\}=c\left\{(n X)^{\alpha} / c\right\}$. This yields $\left\{(n X)^{\alpha}\right\}<1 /(4 a)$. Similarly, $\left\{(n Y)^{\alpha}\right\}<1 /(4 b)$. Further,

$$
\left\{(n Z)^{\alpha}\right\}=\left\{a(n X)^{\alpha} / c+b(n Y)^{\alpha} / c\right\} \leq a\left\{(n X)^{\alpha} / c\right\}+b\left\{(n Y)^{\alpha} / c\right\}<\frac{1}{2 c} .
$$

Hence

$$
|\delta(n)| \leq a\left\{(n X)^{\alpha}\right\}+b\left\{(n Y)^{\alpha}\right\}+c\left\{(n Z)^{\alpha}\right\}<\frac{1}{4}+\frac{1}{4}+\frac{1}{2}=1 .
$$

Therefore $A_{1} \subseteq A$.

We now evaluate the distribution of $A_{1}$. Let $D_{1}(N)$ be the discrepancy of the sequence $\left((n X)^{\alpha} / c,(n Y)^{\alpha} / c\right)_{N<n \leq 2 N}$. Then (2.1) with $K=\left\lfloor N^{\xi}\right\rfloor$ implies that

$$
D_{1}(N) \ll N^{-\xi}+\sum_{0<\left\|\left(h_{1}, h_{2}\right)\right\|_{\infty} \leq N^{\xi}} \frac{1}{\nu\left(h_{1}, h_{2}\right)}\left|\frac{1}{N} \sum_{N<n \leq 2 N} e\left(L\left(h_{1}, h_{2}\right) n^{\alpha}\right)\right| .
$$


For all $u \in \mathbb{R}$, define $f(u)=L\left(h_{1}, h_{2}\right) u^{\alpha}$. For each $N<u \leq 2 N$,

$$
\left|L\left(h_{1}, h_{2}\right)\right| N^{\alpha-(d+2)} \ll\left|f^{(d+2)}(u)\right| \ll\left|L\left(h_{1}, h_{2}\right)\right| N^{\alpha-(d+2)} .
$$

Therefore Lemma 2.1 with $k=d+2$ yields

$$
\begin{aligned}
& \frac{1}{N} \sum_{N<n \leq 2 N} e\left(L\left(h_{1}, h_{2}\right) n^{\alpha}\right) \\
& \ll\left(\left|L\left(h_{1}, h_{2}\right)\right| N^{\alpha-(d+2)}\right)^{1 /\left(2^{d+2}-2\right)}+\frac{\left(\left|L\left(h_{1}, h_{2}\right)\right| N^{\alpha-(d+2)}\right)^{-1 /\left(2^{d+2}-2\right)}}{N^{1 / 2^{d}}} \\
& \ll\left(L\left(N^{\xi}, N^{\xi}\right) N^{\{\gamma\}-2}\right)^{1 /\left(2^{d+2}-2\right)}+\frac{N^{(2-\{\beta\}+\psi) /\left(2^{d+2}-2\right)}}{N^{1 / 2^{d}}},
\end{aligned}
$$

where in the last inequality we used $\alpha-d<\{\gamma\}$ and $d+2-\alpha<2-\{\beta\}$. By the definition of $\psi$, it follows that $(2-\{\beta\}+\psi) /\left(2^{d+2}-2\right)-1 / 2^{d}=-2 \xi$. Then

$$
\frac{1}{N} \sum_{N<n \leq 2 N} e\left(L\left(h_{1}, h_{2}\right) n^{\alpha}\right) \ll\left((X+Y)^{\gamma} N^{\{\gamma\}-2+\xi}\right)^{1 /\left(2^{d+2}-2\right)}+N^{-2 \xi} .
$$

Therefore, since

$$
\sum_{0<\left\|\left(h_{1}, h_{2}\right)\right\|_{\infty} \leq N^{\xi}} \frac{1}{\nu\left(h_{1}, h_{2}\right)} \ll\left(\log N^{\xi}\right)^{2} \ll_{\xi} N^{\xi /\left(2^{d+2}-2\right)},
$$

we have

$$
D_{1}(N) \ll_{\xi} N^{-\xi}+\left((X+Y)^{\gamma} N^{\{\gamma\}-2+2 \xi}\right)^{1 /\left(2^{d+2}-2\right)} .
$$

Let $E_{1}(N)$ be the right-hand side of (3.12). By the definition of discrepancy,

$$
\frac{\#\left(A_{1} \cap(N, 2 N]\right)}{N}=\frac{1}{16 a b c^{2}}+O_{\xi}\left(E_{1}(N)\right) .
$$

By (3.7), we have

$$
(X+Y)^{\gamma} N^{\{\gamma\}-2+2 \xi} \ll R^{\{\gamma\}-2+2 \xi}(X+Y)^{e} .
$$

Here

$$
\begin{aligned}
e & =\gamma+(\{\gamma\}-2+2 \xi)\left(\frac{\gamma^{2}}{\left(2+\{\beta\}-2^{1-\lfloor\beta\rfloor}\right)(2-\{\gamma\})}+\varepsilon\right) \\
& =\gamma\left(1-\frac{\gamma}{2+\{\beta\}-2^{1-\lfloor\beta\rfloor}}\right)-\varepsilon(2-\{\gamma\})+O(\xi) \\
& \leq \gamma \cdot \frac{2+\{\beta\}-\gamma}{2+\{\beta\}-2^{1-\lfloor\beta\rfloor}}-\varepsilon(2-\{\gamma\})+O(\xi)<0
\end{aligned}
$$

for $\xi$ small enough. This yields

$$
E_{1}(N) \ll_{\xi} R^{-\xi}+R^{(\{\gamma\}-2+2 \xi) /\left(2^{d+2}-2\right)} .
$$


Therefore if $\xi$ is sufficiently small and $R$ is sufficiently large, then

$$
\frac{1}{16 a b c^{2}}+O_{\xi}\left(E_{1}(N)\right) \geq \frac{1}{32 a b c^{2}} .
$$

Hence, in this case, \# $(A \cap(N, 2 N]) \geq \#\left(A_{1} \cap(N, 2 N]\right) \geq N /\left(32 a b c^{2}\right)>0$, which implies that there exists $n_{0} \in A$ satisfying (3.6).

CASE 2. We next discuss the case when (3.9) is false, that is, there exist $h_{1}, h_{2} \in \mathbb{Z}$ with $0<\max \left(\left|h_{1}\right|,\left|h_{2}\right|\right) \leq N^{\xi}$ such that

$$
\left|L\left(h_{1}, h_{2}\right)\right|<N^{-\psi} \text {. }
$$

We observe that $h_{1}$ and $h_{2}$ are non-zero and have opposite signs, since if not, then $1 / c \leq\left|L\left(h_{1}, h_{2}\right)\right|<N^{-\psi}$, which causes a contradiction when $R$ is sufficiently large. Thus we may assume that $h_{1}<0<h_{2}$ by multiplying both sides of (3.14) by $|(-1)|$ if necessary. Let $h_{1}^{\prime}=-h_{1}$ and $\theta=L\left(h_{1}, h_{2}\right) / h_{2}$.

In the case $\theta \geq 0$, let

$$
A_{2}=\left\{n \in\left[1, N^{\psi / \alpha} /(8 b c)^{1 / \alpha}\right] \cap \mathbb{N}: 0 \leq\left\{(n X)^{\alpha} /\left(c h_{2}\right)\right\}<\frac{1}{8 a b c N^{\xi}}\right\}
$$

then $A_{2} \subseteq A$. To see why, suppose $n \in A_{2}$. Then

$$
(n X)^{\alpha} / c=h_{2}\left\lfloor(n X)^{\alpha} /\left(c h_{2}\right)\right\rfloor+h_{2}\left\{(n X)^{\alpha} /\left(c h_{2}\right)\right\},
$$

where the first term is an integer and the second term belongs to $[0,1)$. This yields $\left\{(n X)^{\alpha} / c\right\}=h_{2}\left\{(n X)^{\alpha} /\left(c h_{2}\right)\right\}$. Thus we obtain $0 \leq\left\{(n X)^{\alpha} / c\right\}<$ $1 /(4 a c)$. Further, since

$$
(n Y)^{\alpha} / c=\frac{h_{1}^{\prime}}{c h_{2}}(n X)^{\alpha}+n^{\alpha} \theta=h_{1}^{\prime}\left\lfloor(n X)^{\alpha} /\left(c h_{2}\right)\right\rfloor+h_{1}^{\prime}\left\{(n X)^{\alpha} /\left(c h_{2}\right)\right\}+n^{\alpha} \theta,
$$

$h_{1}^{\prime}\left\lfloor(n X)^{\alpha} /\left(c h_{2}\right)\right\rfloor \in \mathbb{Z}, \quad 0 \leq h_{1}^{\prime}\left\{(n X)^{\alpha} /\left(c h_{2}\right)\right\}+n^{\alpha} \theta<\frac{1}{8 b c}+\frac{1}{8 b c}=\frac{1}{4 b c}$,

we have $\left\{(n Y)^{\alpha} / c\right\}=h_{1}^{\prime}\left\{(n X)^{\alpha} /\left(c h_{2}\right)\right\}+n^{\alpha} \theta$ and $0 \leq\left\{(n Y)^{\alpha} / c\right\}<1 /(4 b c)$. Hence, $A_{2} \subseteq A_{1} \subseteq A$.

We next evaluate the distribution of $A_{2}$. Let $V=N^{\psi / \alpha} /\left(2(8 b c)^{1 / \alpha}\right)$, and $D_{2}(N)$ be the discrepancy of the sequence $\left((n X)^{\alpha} /\left(c h_{2}\right)\right)_{V<n \leq 2 V}$. Then by (2.1) with $K=\left\lfloor N^{2 \xi}\right\rfloor$,

$$
D_{2}(N) \ll \frac{1}{N^{2 \xi}}+\sum_{0<|h| \leq N^{2 \xi}} \frac{1}{|h|}\left|\frac{1}{V} \sum_{V<n \leq 2 V} e\left(\left(h /\left(c h_{2}\right)\right) X^{\alpha} n^{\alpha}\right)\right| .
$$

From Lemma 2.1 with $k=d+2$ we deduce

$$
\begin{aligned}
& D_{2}(N) \ll \frac{1}{N^{2 \xi}} \\
& +\sum_{0<|h| \leq N^{2 \xi}} \frac{1}{|h|}\left(\left(\frac{|h| X^{\alpha}}{c h_{2}} V^{\alpha-d-2}\right)^{1 /\left(2^{d+2}-2\right)}+\frac{\left(\frac{|h| X^{\alpha}}{c h} V^{\alpha-d-2}\right)^{-1 /\left(2^{d+2}-2\right)}}{V^{1 / 2^{d}}}\right) .
\end{aligned}
$$


We see that

$$
\begin{gathered}
\sum_{0<|h| \leq N^{2 \xi}} \frac{1}{|h|}\left(\frac{|h| X^{\alpha}}{c h_{2}} V^{\alpha-d-2}\right)^{1 /\left(2^{d+2}-2\right)} \\
\leq\left(X^{\gamma} V^{\{\gamma\}-2}\right)^{1 /\left(2^{d+2}-2\right)} \cdot 2 \sum_{1 \leq h \leq N^{2 \xi}} h^{-1+1 /\left(2^{d+2}-2\right)} \\
\ll\left(X^{\gamma} V^{\{\gamma\}-2}\right)^{1 /\left(2^{d+2}-2\right)} \cdot N^{2 \xi /\left(2^{d+2}-2\right)} .
\end{gathered}
$$

In addition, since $d-\alpha<0$ and $h_{2} \leq N^{\xi}$, we see that

$$
\begin{gathered}
\sum_{0<|h| \leq N^{2 \xi}} \frac{1}{|h|} \cdot \frac{\left(\frac{|h| X^{\alpha}}{c h_{2}} V^{\alpha-d-2}\right)^{-1 /\left(2^{d+2}-2\right)}}{V^{1 / 2^{d}}} \\
\quad \leq\left(\frac{c h_{2}}{X^{\alpha}}\right)^{1 /\left(2^{d+2}-2\right)} V^{(2+d-\alpha) /\left(2^{d+2}-2\right)-1 / 2^{d}} \cdot 2 \sum_{h=1}^{\infty} h^{-1-1 /\left(2^{d+2}-2\right)} \\
\ll N^{\xi} \cdot V^{1 /\left(2^{d+1}-1\right)-1 / 2^{d}}=N^{\xi} V^{\left(-1+2^{-d}\right) /\left(2^{d+1}-1\right)} .
\end{gathered}
$$

Hence

$$
\begin{aligned}
D_{2}(N) & \ll \frac{1}{N^{2 \xi}}+\left(X^{\gamma} N^{2 \xi} V^{\{\gamma\}-2}\right)^{1 /\left(2^{d+2}-2\right)}+N^{\xi} V^{\left(-1+2^{-d}\right) /\left(2^{d+1}-1\right)} \\
& \ll \frac{1}{N^{2 \xi}}+\left(X^{\gamma} N^{2 \xi+\psi(\{\gamma\}-2) / \gamma}\right)^{1 /\left(2^{d+2}-2\right)}+N^{\xi+\psi\left(-1+2^{-d}\right) /\left(\gamma\left(2^{d+1}-1\right)\right)} .
\end{aligned}
$$

Let $E_{2}(N)$ be the right-hand side. Now by 3.7$)$, we have

$$
X^{\gamma} N^{2 \xi+\psi(\{\gamma\}-2) / \gamma} \ll R^{2 \xi+\psi(\{\gamma\}-2) / \gamma}(X+Y)^{e^{\prime}} .
$$

Here

$$
\begin{aligned}
e^{\prime} & =\gamma+\left(2 \xi+\frac{\psi}{\gamma}(\{\gamma\}-2)\right)\left(\frac{\gamma^{2}}{\left(2+\{\beta\}-2^{1-\lfloor\beta\rfloor}\right)(2-\{\gamma\})}+\varepsilon\right) \\
& =\gamma-\gamma \cdot \frac{2+\{\beta\}-2^{1-\lfloor\beta\rfloor}+O(\xi)}{2+\{\beta\}-2^{1-\lfloor\beta\rfloor}-\varepsilon \cdot \frac{\psi}{\gamma}(2-\{\gamma\})+O(\xi)} \\
& =-\varepsilon \cdot \frac{\psi}{\gamma}(2-\{\gamma\})+O(\xi),
\end{aligned}
$$

where we have used 3.8. This implies that for $\xi$ small enough,

$$
\begin{aligned}
& E_{2}(N) \ll N^{-2 \xi}+\left(R^{2 \xi+\psi(\{\gamma\}-2) / \gamma}(X+Y)^{e^{\prime}}\right)^{1 /\left(2^{d+2}-2\right)} \\
& +N^{\xi+\psi\left(-1+2^{-d}\right) /\left(\gamma\left(2^{d+1}-1\right)\right)} \\
& \ll N^{-2 \xi} \text {. }
\end{aligned}
$$

Therefore, by making $\xi$ smaller and $R$ larger if necessary, we get

$$
\frac{\#\left(A_{2} \cap(V, 2 V]\right)}{V}=\frac{1}{8 a b c N^{\xi}}+O\left(E_{2}(N)\right) \geq \frac{1}{16 a b c N^{\xi}}>0 .
$$


Hence, there exists $n_{0} \in A$ such that

$$
n_{0} \ll_{\varepsilon}\left((X+Y)^{\psi / \alpha}\right)^{\gamma^{2} /\left(\left(2+\{\beta\}-2^{1-\lfloor\beta\rfloor}\right)(2-\{\gamma\})\right)+\varepsilon,}
$$

which implies the inequality (3.6) since $\psi<\alpha$. In the case $\theta<0$, let $\theta^{\prime}=$ $L\left(h_{1}, h_{2}\right) / h_{1}>0$. By switching the roles of $\left(\theta, X^{\alpha}\right)$ and $\left(\theta^{\prime}, Y^{\alpha}\right)$, and by a similar argument to the case $\theta \geq 0$, we also find $n_{0} \in A$ satisfying (3.6).

Lemma 3.4. For all $\alpha>0$ and $X, Y, Z \in \mathbb{N}$, define

$$
\eta(\alpha, X, Y, Z)=\min \left\{\frac{\log \left(\left(\left\lfloor W^{\alpha}\right\rfloor+1\right) W^{-\alpha}\right)}{\log W}: W=X, Y, Z\right\} .
$$

For all $\alpha>0$ and $X, Y, Z \in \mathbb{N}$, if $a\left\lfloor X^{\alpha}\right\rfloor+b\left\lfloor Y^{\alpha}\right\rfloor=c\left\lfloor Z^{\alpha}\right\rfloor$, then for all $\tau \in(\alpha, \alpha+\eta(\alpha, X, Y, Z))$, we have

$$
a\left\lfloor X^{\tau}\right\rfloor+b\left\lfloor Y^{\tau}\right\rfloor=c\left\lfloor Z^{\tau}\right\rfloor .
$$

Proof. The claim is clear since we observe that

$$
\left\lfloor X^{\alpha}\right\rfloor=\left\lfloor X^{\tau}\right\rfloor, \quad\left\lfloor Y^{\alpha}\right\rfloor=\left\lfloor Y^{\tau}\right\rfloor, \quad\left\lfloor Z^{\alpha}\right\rfloor=\left\lfloor Z^{\tau}\right\rfloor
$$

for all $\tau \in(\alpha, \alpha+\eta(\alpha, X, Y, Z))$.

4. Lemmas II. Let $2 \leq \beta<\gamma$, and let $a, b, c \in \mathbb{N}$ as in the previous section. Let $x_{0}>0$ be a large parameter. For each $x \geq x_{0}$, let $K(x) \subseteq \mathbb{N}$ be a non-empty finite set. For each $x \geq x_{0}$ and $z \in K(x)$, let $\theta(x, z)$ and $\ell(x, z)$ be positive real numbers, and define an interval $I(x, z)=(\theta(x, z), \theta(x, z)+$ $\ell(x, z))$. For each $x \geq x_{0}$, define

$$
G_{x}=\bigcup_{z \in K(x)} I(x, z) .
$$

Let us consider the following conditions:

(C1) for all integers $x \geq x_{0}, K(x)$ does not contain any multiples of $x$;

(C2) for all integers $x \geq x_{0}$ and $z \in K(x)$, if $z \neq \max K(x)$, then $z+1 \in$ $K(x)$ or $z+2 \in K(x)$

(C3) there exists $Q_{1}>0$ such that for all $x \geq x_{0}$,

$$
\max \left(\inf \left\{|\beta-\alpha|: \alpha \in G_{x}\right\}, \inf \left\{\left|\gamma+x^{-2}-\alpha\right|: \alpha \in G_{x}\right\}\right) \leq Q_{1} x^{-1} ;
$$

(C4) there exists a real number $\kappa \in(0, \infty) \backslash\{1\}$ such that for all $x \geq x_{0}$ and $z \in K(x)$,

$$
\theta(x, z)=\frac{\log \kappa}{\log (z / x)}+O\left(\frac{1}{x^{2} \log x}\right) ;
$$

(C5) there exist $Q_{2}, Q_{3}>0$ and $q>2$ such that for all $x \geq x_{0}$ and $z \in K(x)$,

$$
Q_{2} x^{-q} \leq \ell(x, z) \leq Q_{3} x^{-\beta} ;
$$

(C6) for all integers $x \geq x_{0}, G_{x} \subseteq\left(\beta, \gamma+x^{-2}\right)$; 
(C7) for all integers $x \geq x_{0}$ and $z \in K(x)$, there exists a pairwise distinct tuple $(X(x, z), Y(x, z), Z(x, z)) \in \mathbb{N}^{3}$ such that for all $\tau \in I(x, z)$,

$$
a\left\lfloor X(x, z)^{\tau}\right\rfloor+b\left\lfloor Y(x, z)^{\tau}\right\rfloor=c\left\lfloor Z(x, z)^{\tau}\right\rfloor, \quad X(x, z) \geq x .
$$

Proposition 4.1. Suppose that there exist $x_{0}, K(x), \theta(x, z)$, and $\ell(x, z)$ satisfying $(\mathrm{C} 1)$ to $(\mathrm{C} 7)$. Let $q$ be as in (C5). Then

$$
\operatorname{dim}_{\mathrm{H}}(\{\alpha \in[\beta, \gamma]: a x+b y=c z \text { is solvable in } \operatorname{PS}(\alpha)\}) \geq 2 / q .
$$

REMARK 4.2. The idea of the proof of Proposition 4.1 comes from the proof of Jarník's theorem in Falconer's book [Fal14, Theorem 10.3]. Jarník's theorem states that for every $q>2$ the set of $\alpha \in[0,1]$ such that there exist infinitely many $x, z \in \mathbb{N}$ with $|\alpha-z / x| \leq x^{-q}$ has Hausdorff dimension $2 / q$.

The goal of this section is to prove Proposition 4.1. Suppose that there exist $x_{0}, K(x), \theta(x, z)$, and $\ell(x, z)$ satisfying $(\mathrm{C} 1)$ to $(\mathrm{C} 7)$, and choose such $x_{0}, K(x), \theta(x, z)$, and $\ell(x, z)$. Let $Q_{1}, Q_{2}, Q_{3}, \kappa, q$ be as in (C3) to (C5). Let $x_{1}>0$ and $U_{1}>0$ be large parameters depending on $a, b, c, d, \beta, \gamma, Q_{1}, Q_{2}$, $Q_{3}, \kappa, x_{0}, q$. Below we do not indicate the dependence of those parameters. Let $p$ denote a variable running over prime numbers.

LEMMA 4.3. There exists $B_{1}>0$ such that for all $p \geq x_{1}$ and distinct $z, z^{\prime} \in K(p)$, the intervals $I(p, z)$ and $I\left(p, z^{\prime}\right)$ are separated by a gap of at least $B_{1} p^{-1}$ if $x_{1}$ is sufficiently large.

Proof. By (C4) and (C6), for all $p \geq x_{1}$ and $z \in K(p)$, we have

$$
\frac{\beta}{2} \leq \frac{\log \kappa}{\log (z / p)} \leq 2 \gamma
$$

if $x_{1}$ is sufficiently large. This implies that

$$
p \ll z \ll p .
$$

By (C4) and the inequalities (4.1) and 4.2), there exists $B_{0}>0$ such that

$$
\begin{aligned}
\left|\theta(p, z)-\theta\left(p, z^{\prime}\right)\right| & =\left|\frac{\log \kappa}{\log \frac{z}{p}}-\frac{\log \kappa}{\log \frac{z^{\prime}}{p}}+O\left(\frac{1}{p^{2} \log p}\right)\right| \\
& \geq \frac{|\log \kappa|\left|\log \frac{z^{\prime}}{z}\right|}{\left|\log \frac{z}{p}\right|\left|\log \frac{z^{\prime}}{p}\right|}+O\left(\frac{1}{p^{2} \log p}\right) \\
& \geq \frac{\beta^{2}}{4|\log \kappa|} \log \left(\frac{z+1}{z}\right)+O\left(\frac{1}{p^{2} \log p}\right) \geq B_{0} p^{-1}
\end{aligned}
$$

for all $p \geq x_{1}$ and all $z, z^{\prime} \in K(p)$ with $z<z^{\prime}$. Further, since $\ell(p, z) \leq Q_{3} p^{-2}$ by (C5), there exists $B_{1}>0$ such that for all $p \geq x_{1}$ and distinct $z, z^{\prime} \in K(p)$, the intervals $I(p, z)$ and $I\left(p, z^{\prime}\right)$ are separated by a gap of at least

$$
B_{0} p^{-1}-Q_{3} p^{-2} \geq B_{1} p^{-1}
$$

if $x_{1}$ is sufficiently large. 
Now we call the open interval $I(p, z)(z \in K(p))$ a basic interval of $G_{p}$ for all $p \geq x_{1}$. For each $U \geq U_{1}$, define

$$
H_{U}=\bigcup_{\substack{U<p \leq 2 U \\ p \text { prime }}} G_{p} .
$$

For all $U<p \leq 2 U$, we also call a basic interval of $G_{p}$ a basic interval of $H_{U}$.

LEMMA 4.4. There exist $B_{2}, B_{3}>0$ such that for any $U \geq U_{1}$, all distinct basic intervals of $H_{U}$ are separated by gaps of at least $B_{2} U^{-2}$, and the length of each basic interval of $H_{U}$ is at least $B_{3} U^{-q}$ if $U_{1}$ is sufficiently large.

Proof. We take distinct prime numbers $p$ and $p^{\prime}$ with $U<p, p^{\prime} \leq 2 U$. Then, for all $z \in K(p)$ and $z^{\prime} \in K\left(p^{\prime}\right)$, the condition (C4), the inequality (4.1), and the mean value theorem imply that

$$
\begin{aligned}
\left|\theta(p, z)-\theta\left(p^{\prime}, z^{\prime}\right)\right| & \geq\left|\frac{\log \kappa}{\log (z / p)}-\frac{\log \kappa}{\log \left(z^{\prime} / p^{\prime}\right)}\right|+O\left(\frac{1}{U^{2} \log U}\right) \\
& \geq \frac{\beta^{2}}{4|\log \kappa|}\left|\frac{z}{p}-\frac{z^{\prime}}{p^{\prime}}\right| \min \left(\frac{p}{z}, \frac{p^{\prime}}{z^{\prime}}\right)+O\left(\frac{1}{U^{2} \log U}\right) .
\end{aligned}
$$

We may assume that $p^{\prime} / z^{\prime}>p / z$. By $(\mathrm{C} 1), z$ and $p$ are coprime, which yields $\left|z p^{\prime}-z^{\prime} p\right| \geq 1$. Therefore

$$
\left|\frac{z}{p}-\frac{z^{\prime}}{p^{\prime}}\right| \min \left(\frac{p}{z}, \frac{p^{\prime}}{z^{\prime}}\right)=\left|\frac{z}{p}-\frac{z^{\prime}}{p^{\prime}}\right| \frac{p}{z} \geq \frac{1}{p^{\prime} z} \gg U^{-2}
$$

by the inequalities 4.2 and $U<p, p^{\prime} \leq 2 U$. Therefore for all $U \geq U_{1}$,

$$
\left|\theta(p, z)-\theta\left(p^{\prime}, z^{\prime}\right)\right| \gg U^{-2}
$$

if $U_{1}$ is sufficiently large. Further, for all $U<p \leq 2 U$ and $z \in K(p)$, we deduce by $(\mathrm{C} 5)$ that $\ell(p, z) \ll U^{-\beta}$, where $\beta \geq 2$. Hence there exists $D_{1}>0$ such that for all distinct prime numbers $U<p, p^{\prime} \leq 2 U$, and all $z \in K(p)$ and $z^{\prime} \in K\left(p^{\prime}\right)$, the intervals $I(p, z)$ and $I\left(p^{\prime}, z^{\prime}\right)$ are separated by gaps of at least $D_{1} U^{-2}$. By combining this with Lemma 4.3 , there exists $D_{2}>0$ such that distinct basic intervals of $H_{U}$ are separated by gaps of at least $D_{2} U^{-2}$. Furthermore by (C5), for all $U<p \leq 2 U$ and $z \in K(p)$, we have $Q_{2} \cdot 2^{-q} U^{-q} \leq \ell(p, z)$. In conclusion, we find that all distinct basic intervals of $H_{U}$ are separated by gaps of at least $B_{2} U^{-2}$, and have length of at least $B_{3} U^{-q}$, where we let $B_{2}=D_{2}$ and $B_{3}=Q_{2} \cdot 2^{-q}$.

LEMMA 4.5. There exists $B_{4}>0$ such that the following statement holds: for every $U \geq U_{1}$, if an open interval $I \subset\left(\beta, \gamma+p^{-2}\right)$ satisfies

$$
3 B_{4} / \operatorname{diam}(I)<U<p \leq 2 U,
$$

then the open interval I completely includes at least

$$
\frac{U^{2}}{6 B_{4} \log U} \cdot \operatorname{diam}(I) \quad \text { basic intervals of } H_{U} \text {. }
$$


Proof. By (C4), 4.1), and 4.2), there exists $D_{3}>0$ such that for every $z \in K(p)$ and the minimum $z^{\prime} \in K(p)$ with $z^{\prime}>z$,

$$
\begin{aligned}
\left|\theta(p, z)-\theta\left(p, z^{\prime}\right)\right| & =\left|\frac{\log \kappa}{\log (z / p)}-\frac{\log \kappa}{\log \left(z^{\prime} / p\right)}+O\left(\frac{1}{p^{2} \log p}\right)\right| \\
& \leq \frac{4 \gamma^{2}}{|\log \kappa|} \cdot \frac{1}{z} \cdot\left|z-z^{\prime}\right|+O\left(\frac{1}{p^{2} \log p}\right) \leq D_{3} p^{-1} .
\end{aligned}
$$

Here we apply (C2) when we deduce the last inequality. From (C3), (C6) and (4.7), there exists $B_{4}>0$ such that

$$
\begin{aligned}
\left(\beta, \gamma+p^{-2}\right) \subseteq & \left(\beta, \beta+B_{4} p^{-1}\right) \cup \bigcup_{z \in K(p)}\left(\theta(p, z), \theta(p, z)+B_{4} p^{-1}\right) \\
& \cup\left(\gamma+p^{-2}-B_{4} p^{-1}, \gamma+p^{-2}\right) .
\end{aligned}
$$

Therefore for all $U \geq U_{1}$ and $U<p \leq 2 U$, any open interval $I \subset\left(\beta, \gamma+p^{-2}\right)$ satisfying 4.5 completely includes at least $B_{4}^{-1} p \cdot \operatorname{diam}(I)-2 \geq\left(3 B_{4}\right)^{-1} U$. $\operatorname{diam}(I)$ basic intervals of $G_{p}$. Hence, by the prime number theorem, the open interval $I$ completely includes at least (4.6) basic intervals of $H_{U}$ for a large enough $U_{1}$.

Proof of Proposition 4.1. Let $B_{3}$ and $B_{4}$ be constants as in Lemma 4.4 and Lemma 4.5, respectively. Let $u_{1}=\max \left(U_{1}, 2\right)$. For every $k=2,3, \ldots$, we put

$$
u_{k}=\max \left(u_{k-1}^{k},\left\lceil 3\left(B_{4} / B_{3}\right) u_{k-1}^{q}\right\rceil\right),
$$

and $B_{5}=B_{3} /\left(6 B_{4}\right)$. Let $E_{1}$ be the open interval $(\beta, 2 \gamma)$. For every $k=$ $2,3, \ldots$, let $E_{k}$ be the union of basic intervals of $H_{u_{k}}$ which are completely included by $E_{k-1}$. Let $F$ be the intersection of all $E_{k}$ 's. Define $m_{1}=1$, and for $k \geq 2$, define

$$
m_{k}=\frac{u_{k}^{2}}{6 B_{4} \log u_{k}} B_{3} u_{k-1}^{-q}=B_{5} \frac{u_{k}^{2} u_{k-1}^{-q}}{\log u_{k}} .
$$

Lemma 4.4 implies that each $(k-1)$ st level interval of $F$ has length at least $B_{3} u_{k-1}^{-q}$. Then, by Lemma 4.5 , each $(k-1)$ st level interval of $F$ contains at least $m_{k} k$ th level intervals. In addition, by Lemma 4.4, disjoint $k$ th level intervals of $F$ are separated by gaps of at least $\delta_{k}=B_{2} u_{k}^{-2}$. Therefore, Lemma 2.3 implies that

$$
\begin{aligned}
& \operatorname{dim}_{\mathrm{H}}(F) \geq \varliminf_{k \rightarrow \infty} \frac{\log \left(m_{1} \cdots m_{k-1}\right)}{-\log \left(\delta_{k} m_{k}\right)} \\
& =\varliminf_{k \rightarrow \infty} \frac{2 \log u_{k-1}+\log \left(B_{5}^{k-2} u_{1}^{-q}\left(u_{2} \cdots u_{k-2}\right)^{2-q}\left(\log u_{2}\right)^{-1} \cdots\left(\log u_{k-1}\right)^{-1}\right)}{q \log u_{k-1}+\log \log u_{k}-\log \left(B_{2} B_{5}\right)} .
\end{aligned}
$$

Since $u_{k} \geq u_{k-1}^{k}$ for all $k \geq 2$, we have $\log u_{k} \geq k ! \log u_{1}$ and $u_{k} \geq u_{k-1}$. Further, for $k \geq 1$ large enough, we have $u_{k}=u_{k-1}^{k}$. Thus for $k \geq 1$ large 
enough, we see that

$$
\begin{aligned}
& 2 \log u_{k-1}=2 k^{-1} \log u_{k}, \quad q \log u_{k-1}=q k^{-1} \log u_{k}, \\
& \left|\log \left(B_{5}^{k-2} u_{1}^{-q}\left(u_{2} \cdots u_{k-2}\right)^{2-q}\left(\log u_{2}\right)^{-1} \cdots\left(\log u_{k-1}\right)^{-1}\right)\right| \ll \log u_{k-2} .
\end{aligned}
$$

Therefore, since $\log u_{k-2} / \log u_{k}=1 /(k(k-1)) \rightarrow 0$ as $k \rightarrow \infty$, we get

$$
\operatorname{dim}_{\mathrm{H}}\left(\bigcap_{k=1}^{\infty} E_{k}\right) \geq \frac{2}{q} .
$$

We finally show that for any $\tau \in F$, the equation $a x+b y=c z$ is solvable in $\operatorname{PS}(\tau)$ and $\tau \in[\beta, \gamma]$. If this claim is true, we get the conclusion of Proposition 4.1 by the monotonicity of the Hausdorff dimension.

Take any $\tau \in F$. It is clear that $\tau \in[\beta, \gamma]$ since the condition (C6) yields $H_{u_{k}} \subseteq\left(\beta, \gamma+u_{k}^{-2}\right)$, which implies $F \subseteq[\beta, \gamma]$. Further, by $(\mathrm{C} 7)$, for all $k>1$, there exist a prime number $u_{k}<p_{k} \leq 2 u_{k}$ and $z_{k} \in K\left(p_{k}\right)$ such that we find a pairwise distinct tuple $\left(X\left(p_{k}, z_{k}\right), Y\left(p_{k}, z_{k}\right), Z\left(p_{k}, z_{k}\right)\right) \in \mathbb{N}^{3}$ such that

$$
a\left\lfloor X\left(p_{k}, z_{k}\right)^{\tau}\right\rfloor+b\left\lfloor Y\left(p_{k}, z_{k}\right)^{\tau}\right\rfloor=c\left\lfloor Z\left(p_{k}, z_{k}\right)^{\tau}\right\rfloor, \quad X\left(p_{k}, z_{k}\right) \geq p_{k} .
$$

Since $X\left(p_{k}, z_{k}\right) \geq p_{k} \geq u_{k} \rightarrow \infty$ as $k \rightarrow \infty$, the equation $a x+b y=c z$ is solvable in $\operatorname{PS}(\tau)$.

5. Proof of Theorem 1.1. Fix any $a, b, c \in \mathbb{N}$. Without loss of generality, we may assume that either $a \neq c$ or $a=b=c=1$. Let $\varepsilon>0$ be arbitrarily small. Let $d=\lfloor s\rfloor$ and choose real numbers $\beta, \gamma$ with $d \leq s<$ $\beta<\gamma<\min (t, d+1)$. Let $x_{0}=x_{0}(a, b, c, d, \beta, \gamma)$ be as in Section 3. By the monotonicity of the Hausdorff dimension, we have

$$
\begin{aligned}
\operatorname{dim}_{\mathrm{H}}(\{\alpha \in[s, t]: a x+b y=c z \text { is solvable in } \operatorname{PS}(\alpha)\}) \\
\geq \operatorname{dim}_{\mathrm{H}}(\{\alpha \in[\beta, \gamma]: a x+b y=c z \text { is solvable in } \operatorname{PS}(\alpha)\}) .
\end{aligned}
$$

Take $\alpha(x, z)$ as in Lemmas 3.1 and 3.2 . Let $K(x)=J_{a, b, c}(x), \theta(x, z)=$ $\alpha(x, z)$. We give $\ell(x, z)$ later. Let us check the conditions (C1) to (C7), and apply Proposition 4.1 .

CASE $a>c$. By Lemma 3.1, for all $x \geq x_{0}$ and $z \in J_{a, b, c}(x)$ we have

$$
a x^{\alpha(x, z)}+b=c z^{\alpha(x, z)} \text {. }
$$

Thus by Lemma 3.3, there exists $n_{0}=n_{0}(x, z) \in \mathbb{N}$ such that

$$
\begin{aligned}
& a\left\lfloor\left(n_{0} x\right)^{\alpha}\right\rfloor+b\left\lfloor n_{0}^{\alpha}\right\rfloor=c\left\lfloor\left(n_{0} z\right)^{\alpha}\right\rfloor, \\
& \max \left(\left\{\left(n_{0} x\right)^{\alpha}\right\},\left\{\left(n_{0}\right)^{\alpha}\right\},\left\{\left(n_{0} z\right)^{\alpha}\right\}\right)<1 / 2, \\
& n_{0} \ll_{\varepsilon} x^{\gamma^{2} /\left(\left(2+\{\beta\}-2^{1-\lfloor\beta\rfloor}\right)(2-\{\gamma\})\right)+\varepsilon} .
\end{aligned}
$$

Define $\eta$ as in Lemma 3.4. Let $\ell(x, z)=\eta\left(\alpha(x, z), n_{0} x, n_{0}, n_{0} z\right)$. The condition $(\mathrm{C} 1)$ is clear from the definition of $J_{a, b, c}(x)$. The condition (C2) is also clear since we find at most one multiple of $x$ among any three consecutive 
integers if $x_{0} \geq 3$. Lemma 3.1 implies (C4). By Lemma 3.4, for each $x \geq x_{0}$ and $z \in J_{a, b, c}(x)$, each $\tau \in(\alpha(x, z), \alpha(x, z)+\ell(x, z))$ satisfies

$$
a\left\lfloor\left(n_{0} x\right)^{\tau}\right\rfloor+b\left\lfloor n_{0}^{\tau}\right\rfloor=c\left\lfloor\left(n_{0} z\right)^{\tau}\right\rfloor, \quad n_{0} x \geq x .
$$

Therefore we have (C7). Let us prove (C3), (C5), (C6).

We show (C3). Let $x$ be an integer with $x \geq x_{0}$. For each $i \in\{1,2\}$, let

$$
z_{1, i}=\left\lfloor\left(\frac{b}{c x^{2} \log x}+\frac{a}{c}\right)^{1 / \gamma} x\right\rfloor+i, \quad z_{2, i}=\left\lfloor(a / c)^{1 / \beta} x\right\rfloor-i .
$$

Note that $J_{a, b, c}(x)$ does not contain multiples of $x$. Thus we do not know whether $z_{1, i}, z_{2, i} \in J_{a, b, c}(x)$ for each $i \in\{1,2\}$. However, by (C2), there exist $i_{1}, i_{2} \in\{1,2\}$ such that $z_{1, i_{1}}, z_{2, i_{2}} \in J_{a, b, c}(x)$. Lemma 3.1 implies that

$$
\alpha\left(x, z_{1, i_{1}}\right)=\frac{\log (a / c)}{\log \left(z_{1, i_{1}} / x\right)}+O\left(\frac{1}{x^{2} \log x}\right) .
$$

Here we have

$$
\begin{aligned}
\log \left(z_{1, i_{1}} / x\right) & =\log \left(\left(\frac{b}{c x^{2} \log x}+\frac{a}{c}\right)^{1 / \gamma}+O\left(x^{-1}\right)\right) \\
& =\frac{1}{\gamma} \log (a / c)+\log \left(1+O\left(\frac{b}{a \gamma x^{2} \log x}\right)+O\left(x^{-1}\right)\right) \\
& =\frac{1}{\gamma} \log (a / c)+O\left(x^{-1}\right) .
\end{aligned}
$$

Therefore

$$
\alpha\left(x, z_{1, i_{1}}\right)=\frac{\log (a / c)}{\frac{1}{\gamma} \log (a / c)+O\left(x^{-1}\right)}+O\left(\frac{1}{x^{2} \log x}\right)=\gamma+O\left(x^{-1}\right) .
$$

Similarly, we have $\alpha\left(x, z_{2, i_{2}}\right)=\beta+O\left(x^{-1}\right)$. Hence we obtain (C3).

We next show (C5). For all $x \geq x_{0}$ and $z \in J_{a, b, c}(x)$, we have $x<z$ by the definition of $J_{a, b, c}(x)$. Recall that

$$
\ell(x, z)=\eta\left(\alpha(x, z), n_{0} x, n_{0}, n_{0} z\right)=\frac{\log \left(\left(\left\lfloor W^{\alpha}\right\rfloor+1\right) W^{-\alpha}\right)}{\log W},
$$

where $W$ is one of $n_{0} x, n_{0}$, or $n_{0} z$. From $\beta<\alpha(x, z)$, we have $\ell(x, z) \leq$ $\log \left(1+\left(n_{0} x\right)^{-\beta}\right) \leq x^{-\beta}$. Further, by the facts (5.3), (5.4), $1<x<z \ll x$, and $\alpha<\gamma$, we have

$$
\ell(x, z) \geq \frac{\log \left(1+2^{-1} W^{-\alpha}\right)}{\log W} \gg \frac{1}{\left(n_{0} z\right)^{\gamma} \log \left(n_{0} z\right)} \gg_{\varepsilon} x^{-q},
$$

where

$$
q=q(\beta, \gamma, \varepsilon)=(\gamma+\varepsilon)\left(\frac{\gamma^{2}}{\left(2+\{\beta\}-2^{1-\lfloor\beta\rfloor}\right)(2-\{\gamma\})}+1+\varepsilon\right) .
$$


Therefore (C5) holds (with $Q_{3}=1$ ). The remaining condition (C6) is clear since $\beta<\alpha(x, z)<\gamma$ and $\alpha(x, z)+\ell(x, z)<\gamma+x^{-2}$ by (C5) (with $Q_{3}=1$ ).

CASE $c>a$. Define $n_{0}=n_{0}(x, z)$ and $\ell(x, z), q(\beta, \gamma, \varepsilon)$ the same way as in Case $a>c$. The condition (C1) is clear since $z<x$ by the definition of $J_{a, b, c}(x)$. The condition (C2) is also clear since $J_{a, b, c}(x)$ forms a set of consecutive integers. Lemma 3.1 implies (C4). Similarly to the discussion in Case $a>c$, we have (C5)-(C7). To show (C3), let $x$ be an integer with $x \geq x_{0}$. Let

$$
z_{1}=\left\lfloor\left(\frac{a}{c-b\left(x^{2} \log x\right)^{-1}}\right)^{1 / \beta} x\right\rfloor+1, \quad z_{2}=\left\lfloor(a / c)^{1 / \gamma} x\right\rfloor-1 .
$$

We observe that $z_{1}, z_{2} \in J_{a, b, c}(x)$ if $x_{0}$ is sufficiently large. Lemma 3.1 implies that $\alpha\left(x, z_{1}\right)=\beta+O\left(x^{-1}\right)$ and $\alpha\left(x, z_{2}\right)=\gamma+O\left(x^{-1}\right)$. This gives (C3).

CASE $a=b=c=1$. By Lemma 3.2, for all $x \geq x_{0}$ and $z \in J_{1,1,1}(x)$, by letting $X=X(x, z)=x^{2}\lceil\log x\rceil, Y=Y(x, z)=x^{2}\lceil\log x\rceil+1, Z=$ $Z(x, z)=z x\lceil\log x\rceil$, we have

$$
X^{\alpha(x, z)}+Y^{\alpha(x, z)}=Z^{\alpha(x, z)} .
$$

Therefore, from Lemma 3.3 , there exists $n_{0}=n_{0}(x, z) \in \mathbb{N}$ such that

$$
\begin{aligned}
& \left\lfloor\left(n_{0} X\right)^{\alpha}\right\rfloor+\left\lfloor\left(n_{0} Y\right)^{\alpha}\right\rfloor=\left\lfloor\left(n_{0} Z\right)^{\alpha}\right\rfloor, \\
& \max \left(\left\{\left(n_{0} X\right)^{\alpha}\right\},\left\{\left(n_{0} Y\right)^{\alpha}\right\},\left\{\left(n_{0} Z\right)^{\alpha}\right\}\right)<1 / 2, \\
& n_{0} \ll_{\varepsilon}(X+Y)^{\gamma^{2} /\left(\left(2+\{\beta\}-2^{1-\lfloor\beta\rfloor}\right)(2-\{\gamma\})\right)+\varepsilon} .
\end{aligned}
$$

Defining $r=r(\gamma, \beta, \varepsilon)=\gamma^{2} /\left(\left(2+\{\beta\}-2^{1-\lfloor\beta\rfloor}\right)(2-\{\gamma\})\right)+\varepsilon$, we obtain

$$
n_{0} \ll_{\varepsilon} x^{(2+\varepsilon) r} \text {. }
$$

Let $\ell(x, z)=\eta\left(\alpha(x, z), n_{0} X, n_{0} Y, n_{0} Z\right)$ be as in Lemma 3.4 .

The condition (C1) is clear since $x<z<2 x$ by the definition of $J_{1,1,1}(x)$. The condition (C2) is also clear since $J_{1,1,1}(x)$ forms a set of consecutive integers. Lemma 3.2 implies (C4). By Lemma 3.4 for all $x \geq x_{0}$ and $z \in$ $J_{1,1,1}(x)$, each $\tau \in(\alpha(x, z), \alpha(x, z)+\ell(x, z))$ satisfies

$$
\left\lfloor\left(n_{0} X\right)^{\tau}\right\rfloor+\left\lfloor\left(n_{0} Y\right)^{\tau}\right\rfloor=\left\lfloor\left(n_{0} Z\right)^{\tau}\right\rfloor, \quad n_{0} X \geq x .
$$

Therefore (C7) holds. It remains to prove (C3), (C5), and (C6).

Let us show (C3). Take any integer $x \geq x_{0}$. Let

$$
z_{1}=\left\lfloor 2^{1 / \gamma}\left(x+(x\lceil\log x\rceil)^{-1}\right)\right\rfloor+1, \quad z_{2}=\left\lfloor 2^{1 / \beta} x\right\rfloor-1 .
$$

It follows that $z_{1}, z_{2} \in J_{1,1,1}(x)$ if $x_{0}$ is sufficiently large. Then Lemma 3.2 implies that $\alpha\left(x, z_{1}\right)=\gamma+O\left(x^{-1}\right)$ and $\alpha\left(x, z_{2}\right)=\beta+O\left(x^{-1}\right)$. Therefore we have (C3). 
We next show (C5). Let $x$ be an integer with $x \geq x_{0}$ and $z \in J_{1,1,1}(x)$. It is clear that $x<z$ and $X(x, z)<Y(x, z)<Z(x, z)$. Recall that

$$
\ell(x, z)=\eta\left(\alpha(x, z), n_{0} X, n_{0} Y, n_{0} Z\right)=\frac{\log \left(\left(\left\lfloor W^{\alpha}\right\rfloor+1\right) W^{-\alpha}\right)}{\log W},
$$

where $W$ is one of $n_{0} X, n_{0} Y$, or $n_{0} Z$. Therefore, as $\beta<\alpha$, we have $\ell(x, z) \leq$ $\log \left(1+\left(n_{0} Z\right)^{-\beta}\right) \leq Z^{-\beta} \leq x^{-\beta}$. Further, from (5.5), (5.6) and $\alpha<\gamma$, we obtain

$$
\ell(x, z) \geq \frac{\log \left(1+2^{-1} W^{-\alpha}\right)}{\log W} \gg \frac{1}{\left(n_{0} Z\right)^{\gamma} \log \left(n_{0} Z\right)} \gg_{\varepsilon} x^{-(2+\varepsilon)(\gamma+\varepsilon)(r+1)} .
$$

Hence, (C5) holds. The condition (C6) is clear since $\beta<\alpha(x, z)<\gamma$ and $\alpha(x, z)+\ell(x, z)<\gamma+x^{-2}$ by $(\mathrm{C} 5)$.

To summarize the above discussion, define

$$
D_{a, b, c}(\beta, \gamma, \varepsilon)= \begin{cases}\frac{2}{(2+\varepsilon)(\gamma+\varepsilon)(r(\beta, \gamma, \varepsilon)+1)} & \text { if } a=b=c, \\ \frac{2}{q(\beta, \gamma, \varepsilon)} & \text { otherwise. }\end{cases}
$$

Cases $a>c, c>a, a=b=c=1$ and Proposition 4.1 imply that

$\operatorname{dim}_{\mathrm{H}}(\{\alpha \in[\beta, \gamma]: a x+b y=c z$ is solvable in $\operatorname{PS}(\alpha)\}) \geq D_{a, b, c}(\beta, \gamma, \varepsilon)$.

Therefore, by (5.1) and by letting $\varepsilon \rightarrow+0, \gamma \rightarrow \beta, \beta \rightarrow s$, we have

$\operatorname{dim}_{\mathrm{H}}(\{\alpha \in[s, t]: a x+b y=c z$ is solvable in $\operatorname{PS}(\alpha)\}) \geq D_{a, b, c}(s, s, 0)$.

By the definitions of $q$ and $r$, we get the conclusion of Theorem 1.1 .

Acknowledgements. We would like to thank the referee for valuable comments. The first author was supported by JSPS KAKENHI Grant Number JP20K14292. The second author was supported by JSPS KAKENHI Grant Number JP19J20878.

\section{References}

[DT97] M. Drmota and R. F. Tichy, Sequences, Discrepancies and Applications, Lecture Notes in Math. 1651, Springer, Berlin, 1997.

[Fal14] K. Falconer, Fractal Geometry. Mathematical Foundations and Applications, 3rd ed., Wiley, Chichester, 2014.

[FW09] N. Frantzikinakis and M. Wierdl, A Hardy field extension of Szemerédi's theorem, Adv. Math. 222 (2009), 1-43.

[Gla17] D. Glasscock, Solutions to certain linear equations in Piatetski-Shapiro sequences, Acta Arith. 177 (2017), 39-52.

[Gla20] D. Glasscock, A perturbed Khinchin-type theorem and solutions to linear equations in Piatetski-Shapiro sequences, Acta Arith. 192 (2020), 267-288. 
[Kok50] J. F. Koksma, Some theorems on Diophantine inequalities, Scriptum no. 5, Math. Centrum Amsterdam, 1950.

[SY19] K. Saito and Y. Yoshida, Arithmetic progressions in the graphs of slightly curved sequences, J. Integer Seq. 22 (2019), no. 2, art. 19.2.1, 25 pp.

[Sze75] E. Szemerédi, On sets of integers containing no $k$ elements in arithmetic progression, Acta Arith. 27 (1975), 199-245.

[Szü52] P. Szüsz, Über ein Problem der Gleichverteilung, in: Comptes Rendus du Premier Congrès des Mathématiciens Hongrois, 27 Août - 2 Septembre 1950, Akadémiai Kiadó, Budapest, 1952, 461-472.

[Tit86] E. C. Titchmarsh, The Theory of the Riemann Zeta-Function, 2nd ed., Clarendon Press, New York, 1986.

Toshiki Matsusaka

Institute for Advanced Research

Nagoya University

Furo-cho, Chikusa-ku

Nagoya, 464-8602, Japan

E-mail: matsusaka.toshiki@math.nagoya-u.ac.jp
Kota Saito

Graduate School of Mathematics

Nagoya University

Furo-cho, Chikusa-ku

Nagoya, 464-8602, Japan

E-mail: m17013b@math.nagoya-u.ac.jp 